\title{
Intraocular sarcoidosis: association of clinical characteristics of uveitis with positive chest high-resolution computed tomography findings
}

D.S. Clement ${ }^{1}$, G. Postma' ${ }^{2}$, A. Rothova' ${ }^{2}$, J.C. Grutters ${ }^{3,4}$, M. Prokop ${ }^{1}$, P.A. de Jong ${ }^{1}$

Department of Radiology ${ }^{1}$, Opthalmology ${ }^{2}$ and Pulmonology ${ }^{3}$, University Medical Center Utrecht, Utrecht and department of Pulmonology ${ }^{4}$, St. Antonius Hospital, Nieuwegein, The Netherlands

Correspondence:

Pim A de Jong, MD PhD

University Medical Centre Utrecht

Department of Radiology, HP E.01.132

Heidelberglaan 100, 3584 CX Utrecht

E-mail: pimdejong@gmail.com

Word count manuscript: 2110 


\section{Abstract}

Aim: To assess specific clinical criteria in patients with uveitis that are related to signs of sarcoidosis on high-resolution computed tomography (HRCT) of the chest.

Methods: Retrospective study of 50 consecutive patients with uveitis who were referred for chest HRCT because of suspicion of sarcoidosis. Clinical characteristics, laboratory findings, chest radiographs and chest HRCT scans were retrieved. HRCT scans were re-assessed for signs of sarcoidosis. Mann-Whitney and Fisher's exact test were used for data analysis.

Results: Ten of $50(20 \%)$ uveitis patients referred for HRCT demonstrated signs of sarcoidosis on HRCT. The median age of these patients was significantly higher than those patients with a negative HRCT (71.1 versus 44.7 years, $p=0.002)$. The presence of peripheral chorioretinal punched out lesions and posterior synechiae were significantly related to an abnormal HRCT scan.

Conclusion: Increasing age, presence of peripheral multifocal chorioretinitis and posterior synechiae were associated with an abnormal HRCT scan.

Key-words: uveitis, sarcoidosis, computed tomography, multifocal chorioretinitis Word count: 149 


\section{Introduction}

Sarcoidosis is a relatively common systemic disease with a life-time risk of $0.85 \%$ for Whites and $2.4 \%$ for Blacks in United States. ${ }^{1}$ The cause of sarcoidosis is unknown and a histological proof is required for the definitive diagnosis. The most commonly affected organs are mediastinal and hilar lymph nodes (50-80\%) and the lungs (20-50\%). Respiratory tract involvement occurs in nearly all sarcoidosis patients. ${ }^{1 ; 2}$ Skin and eyes are also frequently involved. ${ }^{3}$ Uveitis is present in approximately $25 \%$ of sarcoidosis patients depending on the population studied. ${ }^{4-6}$

The correct and timely diagnosis of sarcoidosis in uveitis patients is important since it determines the treatment strategy in this often chronic and potentially blinding ocular disorder. Suspicion of ocular sarcoidosis in patients with uveitis is usually based on clinical findings and is further supported by laboratory (serum ACE) and radiological tests (chest $\mathrm{X}$ ray, CXR; high-resolution computed tomography, HRCT); if possible biopsy is taken from affected tissues. The superiority of HRCT over CXR for detecting signs of sarcoidosis is well established but HRCT comes with the disadvantages of a higher radiation dose and higher costs. $^{7-10}$ The value of HRCT of the chest in the diagnostic workup of ocular sarcoidosis is not yet known, and it remains uncertain which patients to refer for HRCT evaluation.

In the present study we assessed which clinical and laboratory characteristics in patients with uveitis are related to signs of sarcoidosis on high-resolution computed tomography $(\mathrm{HRCT})$ of the chest

\section{Methods}

In the present study we included all 50 chest HRCT scans of patients with uveitis and a suspicion of sarcoidosis performed between August 2006 and September 2008. The ethical review board of the University Medical Center Utrecht approved the study; informed consent was not required. 
Medical data of these 50 patients were retrospectively reviewed. We registered age, gender, co-morbidity, current treatments, duration of uveitis and various clinical characteristics including location of the uveitis, presence of posterior synechiae, vitritis, papillitis, vasculitis, cystoid macular edema, peripheral multifocal chorioretinitis and intraocular pressure. Anatomical location was classified according to the Standardization of Uveitis Nomenclature (SUN) criteria. ${ }^{11}$ Intraocular pressure above 22 was considered elevated. All patients had been suspected for the diagnosis of sarcoidosis on the base of their ocular manifestations. Due to retrospective character of this study, next to the clinical suspicion of sarcoidosis, no systematic clinical criteria for performing the chest HRCT scans were employed. We also collected chest radiographs obtained within 3 months before the chest HRCT, results of bronchoalveolar lavage (BAL), biopsies and laboratory data (ACE; ESR; c-reactive protein, CRP; leukocyte count). These specific tests were not available for all patients.

All patients underwent chest HRCT scanning following a routine protocol on a 16detector-row CT scanner (MX8000 IDT or Brilliance-16, Philips, Cleveland, OH) or a 64detector-row CT scanner (Brilliance-64, Philips, Cleveland, OH). Inspiratory scans were acquired in a caudocranial direction using $130 \mathrm{mAs}$ and $120 \mathrm{kVp}$ with a slice thickness of $1 \mathrm{~mm}$ (16-detector-row scanners) or $0.9 \mathrm{~mm}$ (64-detector-row scanner) and an increment of $0.7 \mathrm{~mm}$. Intravenous contrast $(90 \mathrm{ml}$ injected at $3 \mathrm{ml} / \mathrm{sec}$, followed by a flush of $25 \mathrm{ml}$ normal saline at $3 \mathrm{ml} / \mathrm{sec}$ ) was given in all patients to optimize visualization of lymph nodes.

The scans were re-evaluated by one observer who did not know the patients' signs and symptoms other than the presence of uveitis. The original HRCT report and other test results were not available to the observer. In case of a different opinion from the original report a specialized chest radiologist was consulted. Criteria that defined HRCT as positive for sarcoidosis were ${ }^{7 ; 12-15}$ : lymph nodes at left and right hilum and mediastinum with a shortaxis diameter greater than $1 \mathrm{~cm}$, peri-lymphatic pulmonary nodules and other parenchymal lung abnormalities characteristic of sarcoidosis ${ }^{16}$. 
Data were analyzed using SPSS 12.0. Characteristics of patients with a positive HRCT were compared to characteristics of patients with a negative HRCT. The MannWhitney-U test was used for continuous variables and the Fisher's exact test for categorical variables. Data are given as mean \pm standard deviation unless indicated otherwise. $P<0.05$ was considered significant.

\section{Results}

General characteristics of the included patients are illustrated in Table 1 and 2. Posterior and panuveitis was present in 43 of the 50 patients. In patients with positive HRCT, all 10 had posterior and panuveitis.

The HRCT scans demonstrated signs of sarcoidosis in 10 patients $(20 \%)$; nine showed only lymphadenopathy (stage I) and one showed lymphadenopathy and lung nodules (stage II). None of these patients had pulmonary complaints. There were no discrepancies between the HRCT re-assessment and the original HRCT report, demonstrating that signs of sarcoidosis were relatively easy to assess with our HRCT protocol. Four of the patients with an abnormal HRCT had a chest radiograph within the 3 months before the HRCT. Only one of these chest radiographs (25\%) was initially reported as being suspicious for sarcoidosis. Among the 40 patients with a normal HRCT 10 received a chest radiograph, two of which were initially reported as showing possible hilar enlargement in one and possible hilar enlargement and interstitial lung disease in the other. In both patients the subsequently ordered HRCT to confirm or exclude the possible CXR findings ruled in both patients the presence of hilar lympheadenopathy and pulmonary disease out.

The characteristics of patients with normal and abnormal HRCT scans are presented in Table 1. The age of the patients with an abnormal HRCT was $69 \pm 17$ (median 71.1) years and with a normal HRCT $47 \pm 19$ (median 44 ) years $(p=0.002)$. Of the 13 female uveitis patients older than 50 years, 6 (46\%) had an abnormal HRCT scan. The age distribution of 
patients with positive and negative scan outcomes is presented in Figure 1. Gender was not significantly associated with an abnormal HRCT. None of the recorded laboratory data was related to an abnormal HRCT. Serum ACE (normal range 7-20 U/L) was within the normal range in $80 \%$ of the patients with an abnormal HRCT and in $90 \%$ of patients with a normal HRCT.

Ophthalmologic signs for patients are presented in Table 2. The presence of peripheral multifocal chorioretinitis $(p=0.01)$ and posterior synechiae $(p=0.05)$ were significantly associated with an abnormal HRCT. Duration of symptoms or other specific ophthalmologic features were not associated with an abnormal HRCT. There were no differences in co-morbidity between the HRCT positive ( $40 \%$ had one or more co-morbidity) and negative groups (42\% had one or more co-morbidity). Seven patients with a positive HRCT were referred to a pulmonologist. In five the diagnosis of sarcoidosis was confirmed by biopsy, in one patient the BAL was suggestive of sarcoidosis and in one patient no further confirmation was attempted. Of the 10 patients with an abnormal HRCT, two patients received systemic treatment with corticosteroids (oral/systemic) and methotrexate (due to severe ocular involvement), while the others were treated locally and/or by periocular steroid injections.

The final diagnosis of 40 patients with a normal HRCT scan included infectious disease $(n=5)$, Harada's disease $(n=2)$, neuromyelitis optica $(n=1)$; in the remainder was the cause not established $(n=32)$.

\section{Discussion}

In our series, elderly age, presence of peripheral multifocal chorioretinitis and posterior synechiae were significantly associated with HRCT findings suggestive of sarcoidosis.

In most studies on ocular sarcoidosis, two age peaks were noted; first between 20 and 30 years (mostly acute form) and around 60 years (chronic form). The affected patients

were predominantly females. ${ }^{6 ; 17 ; 18}$ We found that HRCT scans were predominantly positive in 
elderly patients. Kaiser et $\mathrm{al}^{8}$ also noted that $62 \%$ of 52 consecutive uveitis patients referred for HRCT were older than 60 years. It may be possible that young patients with ocular sarcoidosis are diagnosed by different means and without the use of HRCT (different clinical presentation, positive CXR and /or tissue biopsy). This hypothesis is in accordance with the more frequent prevalence of Lofgren syndrome at young age and its frequent association with various systemic symptoms, pulmonary involvement and concurrent anterior uveitis. ${ }^{19}$ In contrast, sarcoidosis at elderly age was more frequently associated with posterior uveitis and might be accompanied by less frequent pulmonary involvement. ${ }^{20}$

Peripheral punched-out lesions in the retina are characteristic lesions of peripheral multifocal chorioretinitis, an ocular disorder already previously linked to sarcoidosis in elderly female patients. ${ }^{21 ; 22}$ In the present series, the positive HRCT findings were frequently noted in patients with this typical clinical disorder.

Posterior synechaie are typical for chronic anterior uveitis and were frequently found in chronic types of sarcoidosis. ${ }^{23-25}$ Several laboratory tests were reported to be typical for sarcoidosis, including serum ACE and calcium levels. ${ }^{25-27}$ In our series, however, none of the evaluated tests was found to be associated with an abnormal HRCT, which might be due to a limited systemic activity of the disease in patients and/or related to the limited number of patients included.

The value of CXR and HRCT in patients with uveitis has been debated ${ }^{8-10 ; 18}$ and the higher accuracy of HRCT is generally accepted. Kaiser et $a^{8}$ I examined 30 elderly women with chronic iritis, vitritis or choroiditis of unknown cause using HRCT in all and CXR in a subset of 25 patients. Eight of 25 (32\%) had an abnormal CXR, while HRCT was abnormal in 17 of $30(57 \%)$ patients. Chung et al ${ }^{18}$ studied 60 uveitis patients with biopsy proven sarcoidosis, of which 44 underwent chest HRCT and CXR. CXR was abnormal in $22(50 \%)$ and HRCT in 42 (95\%). Of the 60 patients $68 \%$ first presented at the ophthalmology clinic, the others had extra-ocular presenting symptoms. The age peak was in the $6^{\text {th }}$ decade; no gender differences were noted. Our manuscript adds to the paper by Chung et al since we selected patients suspected of sarcoidosis while Chung studied proven sarcoidosis cases 
and in addition differences between European and Chinese populations might exist. As an extension to the worj by Chung et al we studied laboratory tests and specific ophthalmologic signs. Most of our patients did not undergo CXR within 3 months before or after the HRCT and therefore we cannot compare the value of CXR with that of HRCT. The reason for the low number of CXR in our patients is unknown but some patients might have had a radiograph in another hospital before the referral to our ophthalmology department or had a CXR more than 3 months before the HRCT. In addition, some ophthalmologists might prefer HRCT in some patients with a high likelihood of having sarcoidosis.

Despite the higher accuracy, HRCT is not advocated as a primary screening test because of higher costs and higher radiation exposure. ${ }^{8-10 ; 18}$ However, so far, a systematic study comparing the diagnostic value of CXR and HRCT in a large group of patients with uveitis is not available and specific recommendations on the role of HRCT can not be made. For clinical purposes and for a systematic study it would be valuable to recognize specific clinical criteria associated with a high chance of positive HRCT findings. Our study suggests that the presence of peripheral multifocal chorioretinitis and posterior synechiae in an elderly patient are associated with positive HRCT scan findings.

Our study is limited by the fact that we had no systematic criteria to perform the HRCT scan in patients with uveitis. There might have been patients with uveitis that presented to an ophthalmologist in whom other tests (e.g. positive chest radiograph) led to the diagnosis of sarcoidosis without the use of HRCT and others in whom the diagnosis of sarcoidosis might have been missed. A second drawback is the limited number of abnormal HRCT scans, which increases the likelihood of type-Il errors (i.e. not finding significant differences between HRCT positive and HRCT negative groups), while in a larger study the differences might have been significant. Therefore caution is needed with the conclusion that specific tests, for example ACE, are not useful in uveitis patients.

We have shown that elderly patients with uveitis (especially with peripheral multifocal chorioretinitis and posterior synechiae) and without pulmonary symptoms can be diagnosed with sarcoidosis. From the pulmonologist's point of view, there was no indication to treat 
patients with systemic medications. In our opinion, it is important to make the correct diagnosis of sarcoidosis even in patients with mild pulmonary involvement. The potential benefit for the patients includes the empirical knowledge of best treatment regimens, more information on possible future development of the disease, complications and visual prognosis. In addition, it might prevent unnecessary diagnostic procedures in case of development of systemic manifestations. The costs of the examinations need to be compared with the potential benefit for the patients and only a future study, which would systematically compare the outcome of CXRs and CT, might determine the correct diagnostic strategy and the exact role of the CT in diagnosing the patients with uveitis. In conclusion, our study shows that in patients with uveitis an older age, presence of peripheral multifocal chorioretinitis and posterior synechiae increase the probability of HRCT findings suggestive for sarcoidosis. 


\section{Licence for Publication}

The Corresponding Author has the right to grant on behalf of all authors and does grant on behalf of all authors, an exclusive licence (or non exclusive for government employees) on a worldwide basis to the BMJ Publishing Group Ltd to permit this article (if accepted) to be published in BJO and any other BMJPGL products and sublicences such use and exploit all subsidiary rights, as set out in our licence (http://bjo.bmj.com/ifora/licence.pdf).

\section{Competing Interest}

All authors, none declared. 


\section{References}

1. Newman LS, Rose CS, Maier LA. Sarcoidosis. N.Engl.J.Med. 1997;336:1224-34.

2. Rossman MD, Kreider ME. Lesson learned from ACCESS (A Case Controlled Etiologic Study of Sarcoidosis). Proc.Am.Thorac.Soc. 2007;4:453-6.

3. Iannuzzi MC, Rybicki BA, Teirstein AS. Sarcoidosis. N.Engl.J.Med. 2007;357:215365.

4. Behbehani N, JayKrishnan B, Khadadah M et al. Clinical presentation of sarcoidosis in a mixed population in the middle east. Respir.Med. 2007;101:2284-8.

5. Matsuo T, Fujiwara N, Nakata Y. First presenting signs or symptoms of sarcoidosis in a Japanese population. Jpn.J.Ophthalmol. 2005;49:149-52.

6. JAMES DG, ANDERSON R, LANGLEY D et al. OCULAR SARCOIDOSIS. Br.J.Ophthalmol. 1964;48:461-70.:461-70.

7. Hamper UM, Fishman EK, Khouri NF et al. Typical and atypical CT manifestations of pulmonary sarcoidosis. J.Comput.Assist.Tomogr. 1986;10:928-36.

8. Kaiser PK, Lowder CY, Sullivan P et al. Chest computerized tomography in the evaluation of uveitis in elderly women. Am.J.Ophthalmol. 2002;133:499-505.

9. Kosmorsky GS, Meisler DM, Rice TW et al. Chest computed tomography and mediastinoscopy in the diagnosis of sarcoidosis-associated uveitis. Am.J.Ophthalmol. 1998;126:132-4.

10. Birnbaum AD, Fagan BM, Tessler HH et al. Risks of computerized tomography in the evaluation of chronic uveitis. Am.J.Ophthalmol. 2005;139:951-2. 
11. Jabs DA, Nussenblatt RB, Rosenbaum JT. Standardization of uveitis nomenclature for reporting clinical data. Results of the First International Workshop. Am.J.Ophthalmol. 2005;140:509-16.

12. Miller BH, Rosado-de-Christenson ML, McAdams HP et al. Thoracic sarcoidosis: radiologic-pathologic correlation. Radiographics. 1995;15:421-37.

13. Hennebicque AS, Nunes H, Brillet PY et al. CT findings in severe thoracic sarcoidosis. Eur.Radiol. 2005;15:23-30.

14. Lynch JP, III. Computed tomographic scanning in sarcoidosis. Semin.Respir.Crit Care Med. 2003;24:393-418.

15. Padley SP, Hansell DM, Flower CD et al. Comparative accuracy of high resolution computed tomography and chest radiography in the diagnosis of chronic diffuse infiltrative lung disease. Clin.Radiol. 1991;44:222-6.

16. Hansell DM, Milne DG, Wilsher ML et al. Pulmonary sarcoidosis: morphologic associations of airflow obstruction at thin-section CT. Radiology. 1998;209:697-704.

17. Rothova A, Alberts C, Glasius E et al. Risk factors for ocular sarcoidosis. Doc.Ophthalmol. 1989;72:287-96.

18. Chung YM, Lin YC, Liu YT et al. Uveitis with biopsy-proven sarcoidosis in Chinese-a study of 60 patients in a uveitis clinic over a period of 20 years. J.Chin Med.Assoc. 2007;70:492-6.

19. Mana J, Gomez-Vaquero C, Montero A et al. Lofgren's syndrome revisited: a study of 186 patients. Am.J.Med. 1999;107:240-5. 
20. Yanardag H, Pamuk ON. Older sarcoidosis patients: experience of a medical center in Turkey. South Med.J. 2004;97:472-4.

21. Nolle B, Faul S, Jenisch S et al. Peripheral multifocal chorioretinitis with panuveitis: clinical and immunogenetic characterization in older patients. Graefes Arch.Clin.Exp.Ophthalmol. 1998;236:451-60.

22. Lardenoye CW, Van der LA, de Loos WS et al. Peripheral multifocal chorioretinitis: a distinct clinical entity? Ophthalmology. 1997;104:1820-6.

23. Hassenstein A, Bialasiewicz AA, Knospe V et al. [Incidence of ocular manifestations in patients with histologically confirmed systemic sarcoidosis]. Klin.Monatsbl.Augenheilkd. 2003;220:414-7.

24. Rothova A. Ocular involvement in sarcoidosis. Br.J.Ophthalmol. 2000;84:110-6.

25. Bonfioli AA, Orefice F. Sarcoidosis. Semin.Ophthalmol. 2005;20:177-82.

26. Power WJ, Neves RA, Rodriguez A et al. The value of combined serum angiotensinconverting enzyme and gallium scan in diagnosing ocular sarcoidosis. Ophthalmology. 1995;102:2007-11.

27. Obenauf CD, Shaw HE, Sydnor CF et al. Sarcoidosis and its ophthalmic manifestations. Am.J.Ophthalmol. 1978;86:648-55. 
Table 1: Characteristics of patients with a positive versus a negative HRCT scan

\begin{tabular}{|c|c|c|c|}
\hline & $\begin{array}{l}\text { HRCT positive } \\
\text { for sarcoidosis }\end{array}$ & $\begin{array}{l}\text { HRCT negative } \\
\text { for sarcoidosis }\end{array}$ & $\begin{array}{c}\text { Difference between } \\
\text { groups (P-value) }\end{array}$ \\
\hline Sex (number male / female) & $3 / 7$ & $16 / 24$ & 0.72 \\
\hline Age years (median) & 71.1 & 44.7 & 0.002 \\
\hline $\begin{array}{l}\text { Chest X-ray (abnormal / } \\
\text { normal) }\end{array}$ & $1 / 3$ & $2 / 8$ & 1.00 \\
\hline ACE (raised / normal) & $2 / 8$ & $4 / 34$ & 0.28 \\
\hline CRP (raised / normal) & $2 / 4$ & $8 / 21$ & 0.64 \\
\hline ESR (raised / normal) & $6 / 3$ & $13 / 18$ & 0.25 \\
\hline Leukocytes (raised / normal) & $2 / 8$ & $9 / 27$ & 1.00 \\
\hline Serum calcium (raised/normal) & $0 / 6$ & $0 / 10$ & 1.00 \\
\hline \multicolumn{4}{|c|}{$\begin{array}{l}\text { ACE = angiotensin converting enzyme (normal range } 7-20 \mathrm{U} / \mathrm{L}), \mathrm{CRP}=\mathrm{c} \text {-reactive protein } \\
\text { (normal range } 0-10 \mathrm{mg} / \mathrm{L}) \text {, ESR= erythrocyte sedimentation rate }(1-12 \mathrm{~mm} \text { after } 1 \mathrm{~h}) \text {, } \\
\left.\text { leukocytes (normal range } 4.0-10.0 \times 10^{\wedge} 9 / \mathrm{L}\right) \text {. Chest } \mathrm{X} \text {-ray and laboratory parameters were not }\end{array}$} \\
\hline
\end{tabular}


Table 2: Ophthalmologic findings in patients with a positive versus a negative HRCT scan

\begin{tabular}{|lccc|}
\hline & $\begin{array}{c}\text { HRCT positive } \\
\text { for sarcoidosis }\end{array}$ & $\begin{array}{c}\text { HRT negative } \\
\text { for sarcoidosis }\end{array}$ & $\begin{array}{c}\text { Difference } \\
\text { between groups }\end{array}$ \\
Duration uveitis (years, median) & $\mathbf{N = 1 0}$ & $\mathbf{N}=39 *$ & P-value \\
Unilateral involvement & 1.09 & 0.72 & 0.60 \\
Anterior segment involvement & $2(20 \%)$ & $11(28 \%)$ & 0.71 \\
Synechiae posterior & $8(80 \%)$ & $25(64 \%)$ & 0.46 \\
Vitritis & $6(60 \%)$ & $7(18 \%)$ & 0.01 \\
Papillitis & $9(90 \%)$ & $32(82 \%)$ & 1.00 \\
Vasculitis & $2(20 \%)$ & $15(38 \%)$ & 0.46 \\
Cystoid macular edema & $3(30 \%)$ & $7(18 \%)$ & 0.41 \\
Peripheral multifocal chorioretinitis & $3(30 \%)$ & $9(23 \%)$ & 0.69 \\
Increased ocular pressure & $5(50 \%)$ & $7(18 \%)$ & 0.05 \\
\hline
\end{tabular}

${ }^{*}$ Characteristics could not be assessed in 1 patient with a negative HRCT scan 
Figure 1: Age distribution for patients with a positive and a negative HRCT scan

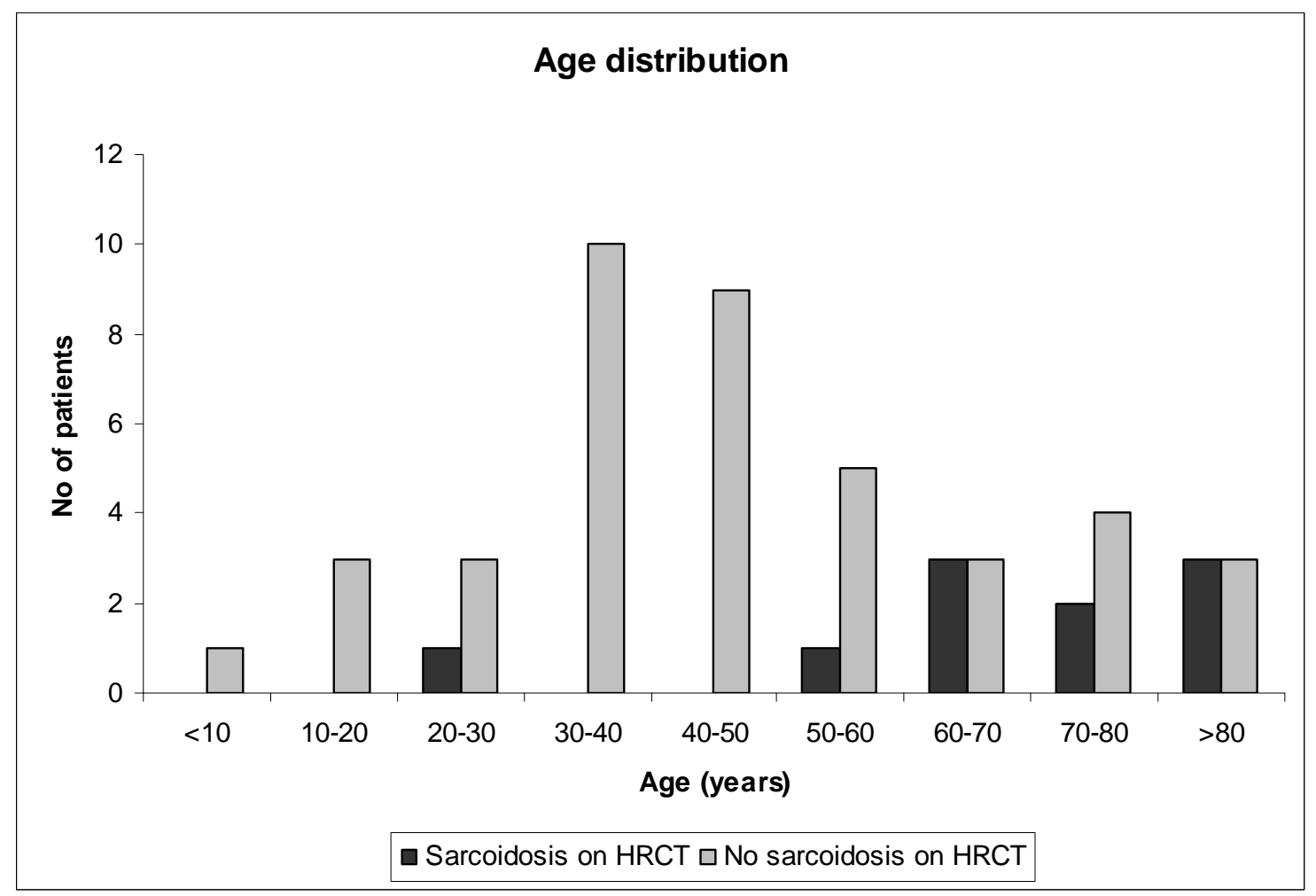

Age distribution differed significantly between the groups, Mann-Whitney $U$ test $p=0.002$. 